Bull. Austral. Math. Soc.

Vol. 74 (2006) [419-441]

\title{
A GENERALISED LUCASIAN PRIMALITY TEST
}

\section{Zhenxiang Zhang, Weiping Zhou and Xianbei LiU}

\begin{abstract}
We present a primality test for numbers of the form $M_{h, n}=h \cdot 2^{n} \pm 1$ (in particular with $h$ divisible by 15), which generalises Berrizbeitia and Berry's test for such numbers with $h \not \equiv 0 \bmod 5$. With our generalised test, the primality of such a number $M_{h, n}$ can be proved by means of a Lucas sequence with a seed determined by $h$ and $\pi_{q}-$ primary irreducible divisor of a prime $q \equiv 1 \bmod 4$. We call the prime $q$ a judge of the number $M_{h, n}$. We prescribe a sequence $\mathcal{S}$ of 48 primes $\equiv 1 \bmod 4$ in the interval $[13,2593]$ such that, for all odd $h=15 t<10^{8}$ and for all $n<7.3 \cdot 10^{11}$, each number $M_{h, n}$ has a judge $q$ in $\mathcal{S}$. Comparisons with Bosma's explicit primality criteria in "a well-defined finite sense" for the case $h=3 t<10^{5}$ are given.
\end{abstract}

\section{INTRODUCTION}

Two classical results express that primality of $2^{n} \pm 1$ can be decided by a single modular exponentiation. Let $n \geqslant 2$, then as Pépin knew in 1877 ,

$$
M=2^{n}+1 \text { is prime } \Longleftrightarrow 3^{(M-1) / 2} \equiv-1 \bmod M .
$$

On the other hand for $2^{n}-1$, the formulation involves a Lucas sequence. The Lucas sequence with seed $w_{0}$ is the sequence $\left\{w_{j}\right\}$ defined from the given initial value $w_{0}$ by the recurrence:

$$
w_{j+1}=w_{j}^{2}-2 \text { for } j \geqslant 0 .
$$

Let $n \geqslant 3$, then as given by Lucas [8] and Lehmer [7],

$$
M=2^{n}-1 \text { is prime } \Longleftrightarrow w_{n-2} \equiv 0 \bmod M,
$$

where $\left\{w_{j}\right\}$ is the Lucas sequence with seed $w_{0}=4$.

The two tests (1.1) and (1.3) generalise to primality tests for integers of the forms

$$
M_{h, n,+}=h \cdot 2^{n}+1 \text { and } M_{h, n,-}=h \cdot 2^{n}-1
$$

Received 5th June, 2006

Research supported by the NSF of China Grant 10071001.

Copyright Clearance Centre, Inc. Serial-fee code: 0004-9727/06 \$A2.00+0.00. 
with $h$ odd and $h<2^{n}$ as follows. (All $h$ in this paper are positive.) As Proth knew in 1878 ,

$$
\begin{aligned}
M=M_{h, n,+} & \text { is prime } \\
& \Longleftrightarrow \text { there exists an integer } b \text { such that } b^{(M-1) / 2} \equiv-1 \bmod M .
\end{aligned}
$$

Whereas for $M_{h, n,-}$, let $d \in \mathbb{Z}$ satisfy

$$
\left(\frac{d}{M}\right)=-1
$$

where $(* / M)$ is the Jacobi symbol. Let $K=\mathbb{Q}(\sqrt{d})$, and let $\mathbb{Z}_{K}$ be the ring of integers of $K$. Let $\alpha \in \mathbb{Z}_{K}$ satisfy

$$
\left(\frac{\alpha \bar{\alpha}}{M}\right)=-1
$$

where $\bar{\alpha}$ denotes the conjugate of $\alpha$ in $K$. Then [10]

$$
M=M_{h, n,-} \text { is prime } \Longleftrightarrow(\alpha / \bar{\alpha})^{(M+1) / 2} \equiv-1 \bmod M \Longleftrightarrow w_{n-2} \equiv 0 \bmod M,
$$

where $\left\{w_{j}\right\}$ is the Lucas sequence with seed

$$
w_{0}=(\alpha / \bar{\alpha})^{h}+(\bar{\alpha} / \alpha)^{h}=\operatorname{Tr}_{K / \mathbb{Q}}(\alpha / \bar{\alpha})^{h} .
$$

Note that, given $h$, the seed $w_{0}$ in (1.9) is uniquely determined by the pair $(d, \alpha)$. For this reason, we call the pair $(d, \alpha)$ satisfying (1.6) and (1.7) (respectively, the integer $b$ in (1.5)) a judge (of primality) of $M_{h, n,-}$ (respectively, of $M_{h, n,+}$ ) for short. In general, the judge depends on $n$ as well as on $h$. However, it is certainly desirable to have it independent of $n$, if possible.

If $h \not \equiv 0 \bmod 3$, one may take $b=3$ in (1.5) and

$$
(d, \alpha)=(12,2+\sqrt{12}), \text { hence } w_{0}=(2+\sqrt{3})^{h}+(2-\sqrt{3})^{h}
$$

in (1.8) for all $n$. More precisely, if

$$
n \geqslant 2 \text { and } 2^{n}>h \neq \equiv \bmod 3,
$$

then [2, Theorem 3.1]

$$
M=M_{h, n,+} \text { is prime } \Longleftrightarrow 3^{(M-1) / 2} \equiv-1 \bmod M ;
$$

and [11, Theorem 4.16], [2, Theorem 3.2]

$$
\begin{aligned}
& M=M_{h, n,-} \text { is prime } \\
& \qquad\left(\frac{2+\sqrt{12}}{2-\sqrt{12}}\right)^{(M+1) / 2} \equiv-1 \bmod M \Longleftrightarrow w_{n-2} \equiv 0 \bmod M,
\end{aligned}
$$


where $\left\{w_{j}\right\}$ is the Lucas sequence with seed $w_{0}$ as given in (1.10).

The case $h \equiv 0 \bmod 3$ is studied in $[2,10]$. In $[10,11]$ tables of seeds are given for $M_{h, n,-}$ for odd $h<30$. In [2] for each $h \equiv 0 \bmod 3, h<10^{5}$, but $h$ not of the form $4^{m}-1$, Bosma designed algorithms for determining finite sets of judges

$$
\mathcal{B}_{h}=\left\{b_{j}\right\}, \text { and } \mathcal{D}_{h}=\left\{\left(d_{j}, \alpha_{j}\right)\right\}
$$

such that, for any $n$, there is a judge of $M_{h, n,+}$ in $\mathcal{B}_{h}$; and a judge of $M_{h, n,-}$ in $\mathcal{D}_{h}$. On the other hand, for $h$ of the form $4^{m}-1$, he proves that there are no such finite sets (1.13) of judges.

Recently, Berrizbeitia and Berry [1] show that, a modification of the test (1.8) allows them, for $h \not \equiv 0 \bmod 5$, to test primality of $M_{h, n,-}$ and $M_{h, n_{1}+}$ by means of a Lucas sequence with a seed independent of $n$. In particular when $h=4^{m}-1, m$ odd, they have a single seed. More precisely, they prove the following Theorem 1, where they use notation

$$
k^{*}=\left(\frac{-1}{k}\right) k
$$

for any odd integer $k$. This notation allows them to treat the cases

$$
M_{h, n}=h \cdot 2^{n} \pm 1
$$

simultaneously, where $M_{h, n}$ means either $M_{h, n,+}$ or $M_{h, n,-}$.

THEOREM 1. ([1, Theorem 3]) Let $M=M_{h, n}=h \cdot 2^{n} \pm 1$ where $h<2^{n-2}-1$ is odd, $h \not \equiv 0 \bmod 5$, and $n \geqslant 4$. Let $\pi=-1+2 i \in \mathbb{Z}[i]$, and let $\left\{w_{j}\right\}$ be the Lucas sequence with seed $w_{0}=(\pi / \bar{\pi})^{h}+(\bar{\pi} / \pi)^{h}$. Then

$$
M \text { is prime } \Longleftrightarrow\left\{\begin{array}{llll}
w_{n-2} \equiv 0 & \bmod M, & \text { when } M^{*} \equiv \pm 2 & \bmod 5 ; \\
w_{n-3} \equiv 0 & \bmod M, & \text { when } M^{*} \equiv-1 & \bmod 5 .
\end{array}\right.
$$

Berrizbeitia and Berry [1] did not say anything about the case $h \equiv 0 \bmod 5$. Of course, if $h \equiv 0 \bmod 5$ but $h \not \equiv 0 \bmod 3$, tests (1.11) and (1.12) are still applicable. The remaining case is therefore $h \equiv 0 \bmod 15$, when it seems that one has to appeal Bosma's approach for determining finite sets (1.13) for the case $h \equiv 0 \bmod 3$.

In this paper, we go a little further to present a generalised test, which includes Berrizbeitia and Berry's test as a special case, and which can treat the cases (1.15) simultaneously as the Berrizbeitia and Berry test does. Moreover, our generalised test treats the case $h \equiv 0$ mod 15 more practically than using Bosma's approach for the case $h \equiv 0 \bmod 3$. We state our generalised test and main tasks of this paper in the following section. 


\section{ThE GENERALISED TEST AND MAIN TASKS}

Let $D=\mathbb{Z}[i]$ be the ring of Gaussian integers, and by a prime we always mean a positive prime of $\mathbb{Z}$. Before stating our generalised test, we recall some basic facts concerning $D$ and biquadratic residue characters. Details can be found in [6, Chapter 9], see also [12, Section 2].

It is well-known that $D$ is a Euclidean domain. Let $\alpha, \beta, \pi \in D$. The norm of $\alpha, N(\alpha)=\alpha \bar{\alpha}=1$ if and only if $\alpha$ is a unit. The units of $D$ are $\pm 1, \pm i$. The irreducibles of $D$ are $\pm 1 \pm i$ with norm 2 , primes $\equiv 3 \bmod 4$ and their associates, and non-real elements with prime norms $\equiv 1 \bmod 4$. A nonunit $\alpha$ is called primary if $\alpha \equiv 1$ or $3+2 i \bmod 4$. Among four associates of a nonunit $\alpha$ satisfying $(1+i) \nmid \alpha$ there is (only) one which is primary. A prime $q \equiv 1 \bmod 4$ must be the norm of a unique primary irreducible $\pi_{q}$ of $D$. If $q$ is small, say $<10^{8}$, then $\pi_{q}$ is easily found by trial and error. There exist efficient algorithms for larger $q$, see [4, Algorithms 2.3.12 and 2.3.13]. If $\pi$ is an irreducible with $N(\pi) \neq 2$, then there exists a unique integer $m, 0 \leqslant m \leqslant 3$, such that $\alpha^{(N(\pi)-1) / 4} \equiv i^{m} \bmod \pi$. The biquadratic residue character of $\alpha$, for $\pi \nmid \alpha$, is defined and denoted by $(\alpha / \pi)_{4}=i^{m}$, which is $1,-1, i$ or $-i$.

Now we are ready to state our generalised test in the following Theorem 2.

THEOREM 2. Let $M=M_{h, n}=h \cdot 2^{n} \pm 1$ where $h<2^{n-2}-1$ is odd and $n \geqslant 4$. Suppose that $q$ is a prime $\equiv 1 \bmod 4$ with primary irreducible $\pi=\pi_{q}=a+b i \in \mathbb{Z}[i]$ satisfying $N(\pi)=\pi \bar{\pi}=q$ and $\left(M^{*} / \pi\right)_{4} \neq 1$. Let $\left\{w_{k}\right\}$ be the Lucas sequence with seed $w_{0}=(\pi / \bar{\pi})^{h}+(\bar{\pi} / \pi)^{h}$. Then

$$
M \text { is prime } \Longleftrightarrow\left\{\begin{array}{lll}
w_{n-2} \equiv 0 & \bmod M, & \text { when }\left(\frac{M^{*}}{\pi}\right)_{4}= \pm i ; \\
w_{n-3} \equiv 0 & \bmod M, & \text { when }\left(\frac{M^{*}}{\pi}\right)_{4}=-1 .
\end{array}\right.
$$

Given $h$ and $n$, let $q$ be a prime $\equiv 1 \bmod 4$. If

$$
\left(\frac{M_{h, \mathrm{n}}^{*}}{\pi_{q}}\right)_{4} \neq 1
$$

then by Theorem 2, the primality of $M_{h, n}$ can be proved by means of a Lucas sequence with seed $w_{0}$ depending only on $\pi_{q}$ and $h$. On the other hand, if

$$
\left\{\begin{array}{l}
\left(\frac{M_{h, n}^{*}}{\pi_{q}}\right)_{4}=1 \\
1<\operatorname{gcd}\left(M_{h, n}, 2^{\text {ord } q}(2)-1\right)<M_{h, n}
\end{array}\right.
$$

or

$$
\left\{\begin{array}{l}
\left(\frac{M_{h, n}^{*}}{\pi_{q}}\right)_{4}=1 \\
1<\operatorname{gcd}\left(M_{h, n}, 2^{\text {ord }(2)}+1\right)<M_{h, n}
\end{array}\right.
$$


then $M_{h, n}$ is a composite, where $\operatorname{ord}_{q}(2)$ denotes the multiplicative order of 2 modulo $q$. Note that $M_{h, n}$ means either $M_{h, n,-}$ or $M_{h, n,+}$.

DEFINITION 2.1: If one of the three equations (2.1), (2.2) and (2.3) holds, we call the prime $q$ a bi-quadratic judge of primality of the number $M_{h, n}$, or a judge of $M_{h, n}$ for short.

DEFINITION 2.2: Given an odd $h$, if there exists a common judge $q$ of the numbers $M_{h, n}$ for all $n$, or in other words, the system

$$
\left\{\begin{array}{l}
\left(\frac{M_{h, n}^{*}}{\pi_{q}}\right)_{4}=1, \\
\operatorname{gcd}\left(M_{h, n}, 2^{\operatorname{ord}_{q}(2)}-1\right)=1=\operatorname{gcd}\left(M_{h, n}, 2^{\operatorname{ord}_{q}(2)}+1\right)
\end{array}\right.
$$

has no solutions in $n$, we call the prime $q$ a minus flag or plus flag of $h$ according to that $M_{h, n}$ means $M_{h, n,-}$ or $M_{h, n,+}$. An odd $h$ may have several minus or plus flags. We denote the smallest minus and plus flag of $h$ by $f_{h}^{-}$and $f_{h}^{+}$respectively.

Not every odd $h=15 t$ has minus and/or plus flags. For those odd $h=15 t$ having no minus and/or plus flags, one wonders whether it would be possible to solve the following problem.

PROBLEM 2.1. Given an odd $h=15 t$. Determine a finite set $\mathcal{W}_{h}^{-}$(respectively $\mathcal{W}_{h}^{+}$) such that for any $n, M_{h, n,-}$ (respectively $M_{h, n,+}$ ) has a judge in $\mathcal{W}_{h}^{-}$(respectively $\mathcal{W}_{h}^{+}$).

In Section 3 we prove Theorem 2, the proof uses biquadratic reciprocity as the proof of Berrizbeitia and Berry's Theorem 1 does. In Section 4, we tabulate 16 primes with the smallest being 13 and the largest being 2089, which are minus or plus flags of some odd $h=15 t<10^{8}$. In Section 5 we prescribe a sequence $\mathcal{S}$ of 48 primes $\equiv 1 \bmod 4$ in the interval $[13,2593]$ having the following properties: for all but a few odd $h=15 t<10^{8}$, there exists a subset (subsequence)

$$
\left.\mathcal{W}_{h}^{-} \text {(respectively } \mathcal{W}_{h}^{+}\right) \subseteq \mathcal{S}
$$

solving Problem 2.1; even if any subset of $\mathcal{S}$ does not solve Problem 2.1 for some odd $h=15 t$ (mainly $h$ is of the form $4^{2 m}-1$ ), that is, none of the elements of $\mathcal{S}$ is a judge of $M_{h, n,-}$ (respectively $M_{h, n,+}$ ) for some $n$, then $n$ would be very large. For this reason, we call $\mathcal{S}$ a universal sequence of judges. Brief conclusions are given in Section 6. Comparisons with Bosma's approach for the case $h \equiv 0 \bmod 3$ are given in relative sections, see Remarks 3.1, 4.1, 5.5 and 5.7.

\section{ProOF OF THEOREM 2}

To prove Theorem 2 we need two lemmas. 
LEMma 3.1. ([1, Corollary 5]) Let $p$ be an odd prime and let $\pi \in \mathbb{Z}[i]$ be primary irreducible. Then

$$
\left(\frac{p^{*}}{\pi}\right)_{4} \equiv(\pi / \bar{\pi})^{\left(p^{*}-1\right) / 4} \bmod p
$$

LEMMA 3.2. ([1, Lemma 7]) Let $p$ be an odd prime and let $\alpha \in \mathbb{Z}[i]$ be prime to $p$. Set $\gamma=\alpha / \bar{\alpha}$. Let $\left\{w_{k}\right\}$ be the Lucas sequence with seed $w_{0}=\operatorname{Tr}(\gamma)=\alpha / \bar{\alpha}+\bar{\alpha} / \alpha$. Suppose that, for some $j, w_{j} \equiv 0 \bmod p$. Then $p \equiv \pm 1 \bmod 2^{j+2}$.

Proof: [Proof of Theorem 2] $(\Longrightarrow)$ Suppose that $M$ is a prime and $\left(M^{*} / \pi\right)_{4} \neq 0,1$. Then $\left(M^{*} / \pi\right)_{4}=-1, \pm i$. By Lemma 3.1,

$$
\left(\frac{M^{*}}{\pi}\right)_{4} \equiv(\pi / \bar{\pi})^{\left(M^{*}-1\right) / 4} \bmod M
$$

If $\left(M^{*} / \pi\right)_{4}=-1$, then $(\pi / \bar{\pi})^{\left(M^{*}-1\right) / 4} \equiv-1 \bmod M$. Thus

$$
w_{n-2}=(\pi / \bar{\pi})^{h \cdot 2^{n-2}}+(\bar{\pi} / \pi)^{h \cdot 2^{n-2}} \equiv-2 \bmod M .
$$

Therefore $w_{n-3} \equiv 0 \bmod M$ follows from the recurrence satisfied be the $w_{j}$.

If $\left(M^{*} / \pi\right)_{4}= \pm i$, Then $(\pi / \bar{\pi})^{\left(M^{*}-1\right) / 4} \equiv \pm i \bmod M$. Thus

$$
w_{n-2}=(\pi / \bar{\pi})^{h \cdot 2^{n-2}}+(\bar{\pi} / \pi)^{h \cdot 2^{n-2}} \equiv 0 \bmod M .
$$

$\left(\Longleftarrow\right.$ Let $p$ be a prime divisor of $M$. Since $w_{n-3} \equiv 0 \bmod M$ or $w_{n-2} \equiv 0 \bmod M$, we have, by Lemma 3.2 ,

$$
p \equiv \pm 1 \bmod 2^{n-1} \text { or } p \equiv \pm 1 \bmod 2^{n} .
$$

Then we have $p \geqslant 2^{n-1}-1$ and $p^{2} \geqslant 2^{2 n-2}-2^{n}+1$. Since

$$
M=h \cdot 2^{n} \pm 1 \leqslant h \cdot 2^{n}+1
$$

and $h<2^{n-2}-1$,

$$
M<2^{2 n-2}-2^{n}+1<p^{2} .
$$

Thus $p>\sqrt{M}$. Therefore $M$ is a prime.

In our generalised test (Theorem 2$)$ we need to check efficiently whether $\left(M_{h, n}^{*} / \pi\right)_{4}$ $\in\{1,-1\}$, hence we use the following Lemma 3.3 and its corollary.

LEMMA 3.3. [12, Lemma 2.5] Let $\pi=u+v i$ be primary irreducible with prime $q=N(\pi) \equiv 1 \bmod 4$ and $\alpha=c+d i$. If $(\alpha / \pi)_{4} \in\{1,-1\}$, then we have

$$
\left(\frac{\alpha}{\pi}\right)_{4} \equiv\left(c-d u v^{-1}\right)^{(q-1) / 4} \bmod q .
$$

NOTE. The condition "If $(\alpha / \pi)_{4} \in\{1,-1\}$ " was missed out in [12, Lemma 2.5] by the author with carelessness while typing. Fortunately, the misprint does not influence any results of [12]). 
Corollary 3.1. Let $\pi=u+v i$ be primary irreducible with prime $q=\pi \bar{\pi}$ $=u^{2}+v^{2} \equiv 1 \bmod 4$. If $\left(M_{h, n}^{*} / \pi\right)_{4} \in\{1,-1\}$, then we have

$$
\begin{aligned}
& \left(\frac{M_{h, n_{1}-}^{*}}{\pi}\right)_{4} \equiv\left(-h_{0} \cdot 2^{n_{0}}+1\right)^{(q-1) / 4} \bmod q ; \\
& \left(\frac{M_{h, n,+}^{*}}{\pi}\right)_{4} \equiv\left(h_{0} \cdot 2^{n_{0}}+1\right)^{(q-1) / 4} \bmod q,
\end{aligned}
$$

where $h_{0}=h \bmod q$ and $n_{0}=n \bmod \operatorname{ord}_{q}(2)$.

EXAMPLE 3.1. Let $N_{n}=85575 \cdot 2^{n}-1$ with $n \geqslant 19$. Then

$$
\left(\frac{N_{n}^{*}}{\pi_{13}}\right)_{4}=1 \Longleftrightarrow N_{n}^{3} \equiv-1 \quad \bmod 13 \Longleftrightarrow n \equiv 1,11 \bmod 12 ;
$$

and

$$
\left(\frac{N_{n}^{*}}{\pi_{17}}\right)_{4}=1 \Longleftrightarrow N_{n}^{4} \equiv 1 \bmod 17 \Longleftrightarrow n \equiv 0,2 \bmod 8 .
$$

Since the system of congruences

$$
\left\{\begin{array}{l}
n \equiv 1,11 \quad \bmod 12 \\
n \equiv 0,2 \bmod 8
\end{array}\right.
$$

has no solutions, 13 is a judge of $N_{n}$ for $n \equiv 0,2,3,4,5,6,7,8,9,10 \bmod 12$ and 17 is a judge of $N_{n}$ for $n \equiv 1,11 \bmod 12$. This means that $\mathcal{W}_{h}^{-}=\{13,17\}$ solves Problem 2.1 for $h=85575=15 \cdot 5705$. Let $\left\{w_{k}\right\}$ be the Lucas sequence with seed

$$
w_{0}=\left(\pi_{13} / \overline{\pi_{13}}\right)^{85575}+\left(\overline{\pi_{13}} / \pi_{13}\right)^{85575}
$$

where $\pi_{13}=3+2 i$, and Let $\left\{w_{k}^{\prime}\right\}$ be the Lucas sequence with seed

$$
w_{0}^{\prime}=\left(\pi_{17} / \overline{\pi_{17}}\right)^{85575}+\left(\overline{\pi_{17}} / \pi_{17}\right)^{85575}
$$

where $\pi_{17}=1+4 i$. Since

$$
\left(\frac{N_{n}^{*}}{\pi_{13}}\right)_{4}=\left\{\begin{array}{l} 
\pm i \Longleftrightarrow n \equiv 0,3,7,8,9,10 \bmod 12 \\
-1 \Longleftrightarrow n \equiv 2,5,6 \bmod 12
\end{array}\right.
$$

and

$$
\left(\frac{N_{n}^{*}}{\pi_{17}}\right)_{4}=\left\{\begin{array}{l} 
\pm i \Longleftrightarrow n \equiv 1,5,6,7 \bmod 8 \\
-1 \Longleftrightarrow n \equiv 3,4 \bmod 8
\end{array}\right.
$$

we have by Theorem $2, N_{n}$ is prime $\Longleftrightarrow$

$$
\left\{\begin{array}{lll}
w_{n-2} \equiv 0 & \bmod N_{n}, & \text { when } n \equiv 0,3,7,8,9,10 \bmod 12 \\
w_{n-3} \equiv 0 & \bmod N_{n}, & \text { when } n \equiv 2,5,6 \bmod 12 \\
w_{n-2}^{\prime} \equiv 0 & \bmod N_{n}, & \text { when } n \equiv 1,13,23 \bmod 24 \\
w_{n-3}^{\prime} \equiv 0 & \bmod N_{n}, & \text { when } n \equiv 11 \bmod 24
\end{array}\right.
$$


Note that, the case $n \equiv 4 \bmod 12$ needs not be tested, since in this case $N_{n}$ is a multiple of 13 . In this way, we find that

$$
\begin{aligned}
& N_{n}(19 \leqslant n \leqslant 1750) \text { is prime } \Longleftrightarrow \\
& n \in\{20,24,26,30,36,42,49,55,60,7072,79,85,90,138,175,209,230,254, \\
& \quad 309,344,373,395,406,479,725,994,1027,1367,1638,1750\} .
\end{aligned}
$$

REMARK 3.1. For testing primality of the numbers $85575 \cdot 2^{n}-1$, Bosma $[2$, Supplement Table 6] used a modulus ('period') $r^{-}=401148$, which is much larger than our modulus 24.

REMARK 3.2. If $\left(M^{*} / \pi\right)_{4}=0$ then either $M=q$ is a prime or $M$ is a multiple of $q$. So, this trivial case is not mentioned in Theorem 2.

REMARK 3.3. If the prime $q$ in Theorem 2 is 5 , then $\pi=\pi_{5}=-1+2 i$. Since

$$
\left(\frac{M^{*}}{\pi}\right)_{4}=1 \Longleftrightarrow h \equiv 0 \bmod 5
$$

Theorem 2 includes Theorem 1 as a special case and $f_{h}^{-}=f_{h}^{+}=5$ for all odd $h \not \equiv 0 \bmod 5$, or in other words, the prime $5=\pi \bar{\pi}$ is a judge of $M_{h, n}$ for all odd $h \not \equiv 0 \bmod 5$ and for any $n \geqslant 4$ with $h<2^{n-2}-1$.

\section{FLAGS OF SOME ODD $h=15 t<10^{8}$}

Let

$$
\mathcal{P}=\left\{\text { prime } p \equiv 1 \bmod 4: 13 \leqslant p<2600, \operatorname{ord}_{p}(2)<350\right\}
$$

Then

$$
\mathcal{P}=\{13,17,29, \ldots, 2441,2593\}
$$

with $\# \mathcal{P}=83$.

In this section we determine the subset $\mathcal{F}$ of $\mathcal{P}$ :

$$
\mathcal{F}=\left\{q \in \mathcal{P}: q \text { is a minus or plus flag of some odd } h=15 t<10^{8}\right\}
$$

For this purpose we need the following lemma, the validity of the lemma is obvious.

LEMMA 4.1. Let prime $q$ be a judge of $M_{h, n}$. If (2.1) or (2.2) holds, then $q$ is a judge of $M_{h^{\prime}, n^{\prime}}$ for all $n^{\prime} \equiv n \bmod \operatorname{ord}_{q}(2)$ and for all odd $h^{\prime} \equiv h \bmod 2^{\text {ord }_{q}(2)}-1$. If (2.3) holds, then $q$ is a judge of $M_{h^{\prime}, n^{\prime}}$ for all $n^{\prime} \equiv n \bmod 2 \operatorname{ord}_{q}(2)$ and for all odd $h^{\prime} \equiv h \bmod 2^{2 \operatorname{ord}_{q}(2)}-1$.

A Pascal (Delphi) program based on Corollary 3.1 and Lemma 4.1 ran about five hours on a PC Pentium III/800 to get the set $\mathcal{F}$, which has 16 elements $q$ tabulated in Table 1, where

$$
\begin{aligned}
& \operatorname{count}_{q}^{-}=\#\left\{\text { odd } h=15 t<10^{8}: f_{h}^{-}=q\right\} \\
& \operatorname{count}_{q}^{+}=\#\left\{\text { odd } h=15 t<10^{8}: f_{h}^{+}=q\right\}
\end{aligned}
$$


$h_{q, 1}^{-}$(respectively, $h_{q, \text { count }}^{-}$) is the smallest (respectively, largest) odd $h=15 t<10^{8}$ with $f_{h}^{-}=q, h_{q, 1}^{+}$(respectively, $h_{q, \text { count } t_{q}^{+}}^{+}$) is the smallest (respectively, largest) odd $h=15 t$ $<10^{8}$ with $f_{h}^{+}=q$.

Out of the 3333333 odd $h=15 t<10^{8}$, there are $\sum$ count $_{q}^{-}=298217$ (about $8.9465 \%$ ) having minus flags $\in \mathcal{P}$, and $\sum$ count $_{q}^{+}=298237$ (about $8.9471 \%$ ) having plus flags $\in \mathcal{P}$.

Table 1: Flags $q$ of some odd $h=15 t<10^{8}$

\begin{tabular}{|r|r|r|r|r|r|r|r|}
\hline$q$ & ord $_{q}(2)$ & count $_{q}^{-}$ & $h_{q, 1}^{-}$ & $h_{q, \text { count }}^{-}$ & count $_{q}^{+}$ & $h_{q, 1}^{+}$ & $h_{q, \text { count }}^{+}$ \\
\hline 13 & 12 & 429 & 296445 & 99814005 & 429 & 97725 & 99976455 \\
37 & 36 & 1 & 37618935 & 37618935 & 2 & 23920125 & 35856285 \\
61 & 60 & 5 & 22464495 & 91298505 & 2 & 31243005 & 69729105 \\
73 & 9 & 234749 & 435 & 99999915 & 234772 & 75 & 99999765 \\
89 & 11 & 16648 & 915 & 99994785 & 16660 & 1425 & 99998865 \\
97 & 48 & 10 & 5180865 & 98908755 & 12 & 6806145 & 93972315 \\
109 & 36 & 2669 & 49485 & 99986685 & 2673 & 123465 & 99978525 \\
241 & 24 & 6755 & 29775 & 99990405 & 6740 & 9315 & 99992625 \\
257 & 16 & 11237 & 10845 & 99988575 & 11237 & 5805 & 99994485 \\
337 & 21 & 25323 & 2115 & 99991725 & 25328 & 1545 & 99999555 \\
433 & 72 & 54 & 184335 & 99835275 & 51 & 313095 & 97758825 \\
601 & 25 & 5 & 9546795 & 90708885 & 5 & 45931785 & 98768385 \\
673 & 48 & 14 & 6821445 & 96863265 & 18 & 11217825 & 94337745 \\
1321 & 60 & 60 & 1196325 & 96400515 & 44 & 2701305 & 98938545 \\
1801 & 25 & 42 & 2327775 & 95816385 & 46 & 220905 & 99560745 \\
2089 & 29 & 216 & 106485 & 99854865 & 218 & 133335 & 99926535 \\
\hline
\end{tabular}

EXAMPLE 4.1. Let $N_{n}=296445 \cdot 2^{n}-1$ with $n \geqslant 21$. Then

$$
\left(\frac{N_{n}^{*}}{\pi_{13}}\right)_{4}=1 \Longleftrightarrow N_{n}^{3} \equiv-1 \bmod 13 \Longleftrightarrow n \equiv 2,4 \bmod 12 \text {. }
$$

Note that $\operatorname{ord}_{13}(2)=12$. We have

$$
\begin{cases}\operatorname{gcd}\left(N_{n}, 2^{12}-1\right)=7 & \text { for } n \equiv 2 \bmod 12 \\ \operatorname{gcd}\left(N_{n}, 2^{12}+1\right)=17 & \text { for } n \equiv 4 \bmod 24 \\ \operatorname{gcd}\left(N_{n}, 2^{12}+1\right)=241 & \text { for } n \equiv 16 \bmod 24\end{cases}
$$

So, 13 is a minus flag of 296445 and thus $f_{296445}^{-}=13$. Let $\left\{w_{k}\right\}$ be the Lucas sequence with seed

$$
w_{0}=(\pi / \bar{\pi})^{296445}+(\bar{\pi} / \pi)^{296445}
$$


where $\pi=\pi_{13}=3+2 i$. Since

$$
\left(\frac{N_{n}^{*}}{\pi}\right)_{4}=\left\{\begin{array}{l} 
\pm i \Longleftrightarrow n \equiv 0,1,3,6,10,11 \bmod 12 \\
-1 \Longleftrightarrow n \equiv 5,8,9 \bmod 12
\end{array}\right.
$$

we have by Theorem 2 ,

$$
N_{n} \text { is prime } \Longleftrightarrow\left\{\begin{array}{lll}
w_{n-2} \equiv 0 & \bmod N_{n}, & \text { when } n \equiv 0,1,3,6,10,11 \bmod 12 \\
w_{n-3} \equiv 0 & \bmod N_{n}, & \text { when } n \equiv 5,8,9 \bmod 12 .
\end{array}\right.
$$

Note that, if $n \equiv 7 \bmod 12$, then $N_{n} \equiv 0 \bmod 13$. In this way, we find that

$N_{n}(21 \leqslant n \leqslant 1750)$ is prime $\Longleftrightarrow$

$$
n \in\{22,34,39,73,78,165,207,333,334,423,613,798,813,819,874\} .
$$

EXAMPLE 4.2. Let $N_{n}=97725 \cdot 2^{n}+1$ with $n \geqslant 19$. Then

$$
\left(\frac{N_{n}^{*}}{\pi_{13}}\right)_{4}=1 \Longleftrightarrow N_{n}^{3} \equiv 1 \quad \bmod 13 \Longleftrightarrow n \equiv 1,11 \bmod 12 \text {. }
$$

We have

$$
\begin{cases}\operatorname{gcd}\left(N_{n}, 2^{12}-1\right)=7 & \text { for } n \equiv 11 \bmod 12, \\ \operatorname{gcd}\left(N_{n}, 2^{12}+1\right)=241 & \text { for } n \equiv 1 \bmod 24, \\ \operatorname{gcd}\left(N_{n}, 2^{12}+1\right)=17 & \text { for } n \equiv 13 \bmod 24 .\end{cases}
$$

So, 13 is a plus flag of 97725 and thus $f_{97725}^{+}=13$. Let $\left\{w_{k}\right\}$ be the Lucas sequence with seed

$$
w_{0}=(\pi / \bar{\pi})^{97725}+(\bar{\pi} / \pi)^{97725}
$$

where $\pi=\pi_{13}=3+2 i$. Since

$$
\left(\frac{N_{n}^{*}}{\pi}\right)_{4}=\left\{\begin{array}{l} 
\pm i \Longleftrightarrow n \equiv 0,3,7,8,9,10 \bmod 12 \\
-1 \Longleftrightarrow n \equiv 2,5,6 \bmod 12
\end{array}\right.
$$

we have by Theorem 2 ,

$$
N_{n} \text { is prime } \Longleftrightarrow\left\{\begin{array}{lll}
w_{n-2} \equiv 0 & \bmod N_{n}, & \text { when } n \equiv 0,3,7,8,9,10 \bmod 12 ; \\
w_{n-3} \equiv 0 & \bmod N_{n}, & \text { when } n \equiv 2,5,6 \bmod 12 .
\end{array}\right.
$$

Note that, if $n \equiv 4 \bmod 12$, then $N_{n} \equiv 0 \bmod 13$. In this way, we find that

$N_{n}(19 \leqslant n \leqslant 1750)$ is prime $\Longleftrightarrow$

$$
n \in\{27,63,91,147,210,282,526,750,1051,1134,1260,1476\} .
$$

REMARK 4.1. Bosma [2] did not give examples of odd $h=3 t<10^{5}$ having "flags" by his approach. We do not know whether there exist odd $h=3 t<10^{5}$ having "flags" by Bosma's approach. 


\section{A UNIVERSAL SEQUENCE OF JUDGES}

Let

$$
\mathcal{S}=\left\{q_{1}, q_{2}, \ldots, q_{s}\right\}
$$

be a finite sequence of different primes $q_{j} \equiv 1 \bmod 4$,

$$
Q=\prod_{q \in \mathcal{S}} q, \text { and let } \mathcal{G}_{Q}=\mathbb{Z}_{Q}^{*}
$$

be the multiplicative group of invertible elements modulo $Q$. Then

$$
\# \mathcal{G}_{Q}=\prod_{q \in \mathcal{S}}(q-1)
$$

Let $\mathcal{H}_{Q}=\langle 2\rangle$ be the cyclic subgroup generated by 2 of $\mathcal{G}_{Q}$. It is clear that if the system of equations

$$
\left(\frac{M_{15 t, n}^{*}}{\pi_{q_{1}}}\right)_{4}=\left(\frac{M_{15 t, n}^{*}}{\pi_{q_{2}}}\right)_{4}=\cdots=\left(\frac{M_{15 t, n}^{*}}{\pi_{q_{t}}}\right)_{4}=1
$$

has solutions in $n$ for a given odd $t=t_{0}$, then it has solutions in $n$ for all odd $t \in t_{0} \mathcal{H}_{Q}$, or in other words, for all odd

$$
t \equiv t_{0} \cdot 2^{m} \bmod Q \text { with } m \geqslant 0 .
$$

Thus we have proved the following lemma concerning the structure of the quotient group $\mathcal{G}_{Q} / \mathcal{H}_{Q}$.

LEMMA 5.1. The quotient group

$$
\mathcal{G}_{Q} / \mathcal{H}_{Q}=\left\{\bar{t}: t \in \mathcal{G}_{Q}\right\}=\mathcal{U}_{Q}^{-} \cup \mathcal{V}_{Q}^{-}=\mathcal{U}_{Q}^{+} \cup \mathcal{V}_{Q}^{+},
$$

where

$$
\left\{\begin{array}{l}
\mathcal{U}_{Q}^{-}=\left\{\bar{t}: \text { (5.3) has solutions in } n \text { for } M_{15 t, n,-}\right\} \\
\mathcal{V}_{\bar{Q}}^{-}=\left\{\bar{t}: \text { (5.3) has no solutions in } n \text { for } M_{15 t, n,-}\right\}
\end{array}\right.
$$

and

$$
\left\{\begin{array}{l}
\mathcal{U}_{Q}^{+}=\left\{\bar{t}: \text { (5.3) has solutions in } n \text { for } M_{15 t, n,+}\right\} \\
\mathcal{V}_{Q}^{+}=\left\{\bar{t}: \text { (5.3) has no solutions in } n \text { for } M_{15 t, n,+}\right\}
\end{array}\right.
$$

Moreover, both maps

$$
\mathcal{U}_{Q}^{-} \rightarrow \mathcal{U}_{Q}^{+} ; \bar{t} \mapsto \overline{Q-t}
$$

and

$$
\mathcal{V}_{Q}^{-} \rightarrow \mathcal{V}_{Q}^{+} ; \bar{t} \mapsto \overline{Q-t}
$$

are bijective. Thus

$$
\# \mathcal{U}_{Q}^{-}=\# \mathcal{U}_{Q}^{+} ; \# \mathcal{V}_{Q}^{-}=\# \mathcal{V}_{Q}^{+}
$$


Put

$$
u=\# \mathcal{U}_{Q}^{-}=\# \mathcal{U}_{Q}^{+} \text {and } v=\# \mathcal{V}_{Q}^{-}=\# \mathcal{V}_{Q}^{+}
$$

Define

$$
\tau_{Q}=\frac{v}{u+v}
$$

$\mathcal{A}^{-}(h, \mathcal{S})=\left\{n: 0 \leqslant n<2 L,(2.4)\right.$ holds for all $q \in \mathcal{S}$ with $\left.M_{h, n}=M_{h, n,-}\right\}$,

$$
\mathcal{A}^{+}(h, \mathcal{S})=\left\{n: 0 \leqslant n<2 L,(2.4) \text { holds for all } q \in \mathcal{S} \text { with } M_{h, n}=M_{h, n,+}\right\},
$$

where

$$
L=\operatorname{Lcm}\left(\operatorname{ord}_{q_{1}}(2), \operatorname{ord}_{q_{2}}(2), \ldots, \operatorname{ord}_{q_{s}}(2)\right)=\# \mathcal{H}_{Q} .
$$

If $\mathcal{A}^{-}(h, \mathcal{S}) \neq \emptyset$ or $\mathcal{A}^{+}(h, \mathcal{S}) \neq \emptyset$, define

$$
\begin{aligned}
& g^{-}(h, \mathcal{S})=\min \left\{n>0: n \in \mathcal{A}^{-}(h, \mathcal{S})\right\}, \\
& g^{+}(h, \mathcal{S})=\min \left\{n>0: n \in \mathcal{A}^{+}(h, \mathcal{S}), M_{h, n,+} \text { is not a perfect square }\right\}
\end{aligned}
$$

We call $\tau_{Q}$ the impact factor of $Q$ or of $\mathcal{S}$. We hope that $\mathcal{S}$ has impact factor close to 1 and has as few elements as possible. If $\mathcal{A}^{+}(h, \mathcal{S}) \neq \emptyset$ (respectively, $\mathcal{A}^{-}(h, \mathcal{S}) \neq \emptyset$ ) for some odd $h=15 t$, we also hope that $g^{+}(h, \mathcal{S})$ (respectively $g^{-}(h, \mathcal{S})$ ) is rather large.

Let $\mathcal{P}$ be as defined by $(4.1)$ and let

$$
\mathcal{S}^{\prime}=\left\{q \in \mathcal{P}: \exists p(\neq q) \in \mathcal{P} \text { such that } \tau_{p q}>0\right\} .
$$

For determining the set $\mathcal{S}^{\prime}$ we need a procedure described in $\S 5.1$ to find $\tau_{p q}$ for given $p, q \in \mathcal{P}$. We find that $\# S^{\prime}=48$ and that $\tau_{13.17}=0.5>\tau_{p q}$ for all $p, q \in \mathcal{P}$ with $\{p, q\} \neq\{13,17\}$. In $\S 5.2$, we sort the elements in the set $\mathcal{S}^{\prime}$ to obtain a universal sequence (5.1) with $q_{1}=13$ and $q_{2}=17$, mainly based on the condition

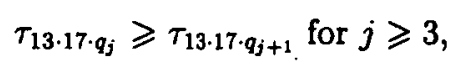

adjusted partially by $\tau_{p q}$ for some $p, q \in \mathcal{S}^{\prime}$. In $\S 5.3$, we show how the universal sequence $\mathcal{S}$ works well for our generalised test.

REMARK 5.1. Note that, the definitions of $\mathcal{U}_{Q}^{-1+}$ and $\mathcal{V}_{Q}^{-1+}$ (see (5.5) and (5.6)) do not involve the gcd condition in (2.4). Since otherwise, the quotient group structure (5.4) would not be valid. But in practical implementation of our generalised test, the gcd checking makes things speed up. See Remark 5.2 below.

Remark 5.2. It is clear that, if $\bar{t} \in \mathcal{V}_{Q}^{-}$, then $\mathcal{W}_{15 t}^{-}=S$ solves Problem 2.1. On the other hand, if $\bar{t} \in \mathcal{U}_{Q}^{-}$, then system (5.3) has solutions in $n$ for this $t$. Suppose $n=n_{0}$ is one of the solutions. If there is some $q \in S$ such that (2.2) or (2.3) holds for $n=n_{0}$, then $M_{15 t, n,-}$ is composite for all $n \equiv n_{0} \bmod _{\operatorname{ord}_{q}}(2)$ or for all $n \equiv n_{0} \bmod 2 \operatorname{ord}_{q}(2)$; otherwise if (2.4) holds for all $q \in \mathcal{S}$, then no subsets of $\mathcal{S}$ solve Problem 2.1 for $h=15 t, \bar{t} \in \mathcal{U}_{Q}^{-}$, thus for testing primality of $M_{15 t, n_{0},-}$, one should look for a judge outside the sequence $\mathcal{S}$. Corresponding words can be said for the case $M_{15 t, n,+}$. 
EXAMPLE 5.1. Note that $\operatorname{ord}_{13}(2)=12$, $\operatorname{ord}_{17}(2)=8$ and $13 \cdot 17=221$. We have

$$
\begin{aligned}
\# \mathcal{G}_{221} & =(13-1) \cdot(17-1)=192, L=\# \mathcal{H}_{221}=\operatorname{Lcm}\left(\operatorname{ord}_{13}(2), \operatorname{ord}_{17}(2)\right)=24, \\
\mathcal{U}_{221} & =\{\overline{1}, \overline{5}, \overline{23}, \overline{55}\}, \mathcal{V}_{221}=\{\overline{3}, \overline{9}, \overline{11}, \overline{25}\}, \quad \text { and } \tau_{221}=\frac{4}{4+4}=0.5 .
\end{aligned}
$$

If $\bar{t} \in \mathcal{V}_{221}$, then $\mathcal{W}_{15 t}^{-}=\{13,17\}$ solves Problem 2.1 for $h=15 t$. Since $3 \cdot 2^{15}$ $\equiv 5705 \bmod 221, \overline{5705}=\overline{3} \in \mathcal{V}_{221}$, thus $\mathcal{W}_{h}^{-}=\{13,17\}$ solves Problem 2.1 for $h=15.5705$ $=85575$, see Example 3.1.

\subsection{Determining the Set $\mathcal{S}^{\prime}$}

Given primes $p, q \equiv 1 \bmod 4$, let

$$
\begin{aligned}
m_{0} & =\# G_{p q}=(p-1)(q-1), m_{1}=\operatorname{Lcm}\left(\operatorname{ord}_{p}(2), \operatorname{ord}_{q}(2)\right), \\
r & =\#\left\{\text { prime } q^{\prime} \equiv 1 \bmod 4: 5<q^{\prime}<q, \# \mathcal{V}_{q^{\prime} q}>0\right\},
\end{aligned}
$$

and let $p_{0}$ be a prime $\equiv 1 \bmod 4$ with impact factor $\tau_{p_{0} q} \geqslant \tau_{q^{\prime} q}$ for all $5<q^{\prime}<q$.

We use the following Procedure 5.1 to find $\tau_{p q}$ and use Procedure 5.2 to determine the set $\mathcal{S}^{\prime}$.

Procedure 5.1. Finding $\tau_{p q}$;

\{input primes $p, q \equiv 1 \bmod 4$ with $p \neq q$; \}

\{output $u$ and $v$ as defined by (5.7) with $Q=p q$, thus $\tau_{p q}=v /(u+v)$;

\{output also $r, m_{0}, m_{1}$ as defined by (5.14) and (5.15) \}

Begin $m_{0} \leftarrow(p-1) \cdot(q-1) ; d \leftarrow \operatorname{gcd}\left(\operatorname{ord}_{p}(2), \operatorname{ord}_{q}(2)\right)$;

$m_{1} \leftarrow \operatorname{Lcm}\left(\operatorname{ord}_{p}(2), \operatorname{ord}_{q}(2)\right) ;$

For $t:=1$ To $p q$ Do

If $(t \equiv 0 \bmod p)$ Or $(t \equiv 0 \bmod q)$ Then $T_{t} \leftarrow$ True Else $T_{t} \leftarrow$ False;

$u \leftarrow 0 ; t \leftarrow 1$;

Repeat $h \leftarrow t \cdot 15$;

Using Corollary 3.1 to find solutions in $n$ of $\left(M_{h, n,-}^{*} / \pi_{p}\right)_{4}=1$ by trial and error; (Suppose $k$ solutions $n=n_{11}, \ldots, n_{1 k} \operatorname{modord}_{p}(2)$ are found)

Using Corollary 3.1 to find solutions in $n$ of $\left(M_{h, n_{-}-}^{*} / \pi_{q}\right)_{4}=1$ by trial and error; (Suppose $l$ solutions $n=n_{21}, \ldots, n_{2 l} \bmod _{\operatorname{ord}}(2)$ are found)

$j \leftarrow 0$; morejudge $\leftarrow$ False;

repeat $j \leftarrow j+1 ; i \leftarrow 0$;

Repeat $i \leftarrow i+1$;

If $\left(n_{2 j}-n_{1 i}\right) \equiv 0 \bmod d$ Then morejudge $\leftarrow$ True

Until morejudge or $(i=k)$

until morejudge or $(j=l)$;

If morejudge Then

begin $x \leftarrow t ; u \leftarrow u+1 ; T_{x} \leftarrow T r u e ; i \leftarrow 0$; 
repeat $x \leftarrow x+x$; If $x \geqslant p q$ Then $x \leftarrow x-p q ; T_{x} \leftarrow$ True; $i \leftarrow i+1$ until $i=m_{1}$

end;

repeat $t \leftarrow t+2$ until $\left(\operatorname{not} T_{t}\right)$ Or $(t=p q)$

Until $t=p q$;

$v \leftarrow 0 ; t \leftarrow 0$;

Repeat repeat $t \leftarrow t+1$ until (not $\left.T_{t}\right)$ or $(t=p q$ );

If ( $t$ is odd) And (not $T_{t}$ ) Then

begin $x \leftarrow t ; v \leftarrow v+1 ; T_{x} \leftarrow$ True; $i \leftarrow 0$;

repeat $x:=x+x$; If $x \geqslant p q$ Then $x \leftarrow x-p q ; T_{x} \leftarrow$ True; $i \leftarrow i+1$

until $i=m_{1}$

end

Until $t=p q$;

$\tau_{p q} \leftarrow v /(u+v)$

End;

Procedure 5.2. Determining the set $\mathcal{S}^{\prime}$;

\{Input the set $\mathcal{P}=\left\{p_{1}, \ldots, p_{83}\right\}$ as defined by (4.1); $\}$

\{Output the set $\mathcal{S}^{\prime}$ as defined by (5.12)\}

Begin Let $\mathcal{S}^{\prime}$ be an empty set; $j \leftarrow 1$;

Repeat $j \leftarrow j+1 ; q \leftarrow p_{j} ; i \leftarrow 0 ; \max \tau \leftarrow 0 ; r \leftarrow 0$;

repeat $i \leftarrow i+1 ; p \leftarrow p_{i}$;

If $\tau_{p q}>0$ Then begin $r \leftarrow r+1 ; \mathcal{S}^{\prime} \leftarrow \mathcal{S}^{\prime} \cup\{p\}$ end;

If $\tau_{p q}>\max \tau$ Then begin $\max \tau \leftarrow \tau_{p q} ; p_{0} \leftarrow p$ end until $i=j-1$

If $r>0$ Then

begin $\mathcal{S}^{\prime} \leftarrow \mathcal{S}^{\prime} \cup\{q\}$

output $\left(q, r, p_{0}\right)$ and related values $\left(m_{0}, m_{1}, u, v, \tau_{q p_{0}}\right)$

(which are found by Procedure 5.1 for finding $\tau_{q p_{0}}$ )

end

Until $j=83$

End.

The Pascal (Delphi) program ran about 3 hours on a PC Pentium III/800 to get 48 elements 
of the set $\mathcal{S}^{\prime}$ with related values tabulated in Table 2. Thus

$$
\begin{aligned}
& \mathcal{S}^{\prime}=\{\text { primes } q \text { in the first column of Table } 2\} \\
& \cup\left\{\text { primes } p_{0} \text { in the third column of Table } 2\right\} \\
& \cup\{\text { primes } p \text { in the last column of Table } 2\} \\
&=\{13,17,29,37,41,53,61,73,89,97,101,109,113,137,157,193,229,233,241, \\
& 257,277,337,349,353,397,401,433,457,577,593,601,641,673,881,937, \\
&953,1013,1217,1249,1321,1429,1613,1657,1777,1801,2089,2113,2593\} .
\end{aligned}
$$

Note that all elements $q$ of the set $\mathcal{F}$ (tabulated in Table 1) are contained in $\mathcal{S}^{\prime}$.

Remark 5.3. By (5.4), if $(u+v) \cdot m_{1} \neq m_{0}$ in the outputs of Procedure 5.1, then there must be some errors in the programming.

5.2. Determining The SEQUence $\mathcal{S}$ Without much modification to Procedure 5.1, we have a procedure for computing $\tau_{13.17 \cdot q}$ for $q \in \mathcal{S}^{\prime} \backslash\{13,17\}$. We sort elements of $\mathcal{S}^{\prime}$ mainly based on (5.13), adjusted partially by $\tau_{p q}$ for some $p, q \in \mathcal{S}^{\prime}$, to obtain the sequence $\mathcal{S}$ tabulated in Table 3 with related values:

$$
\begin{gathered}
\operatorname{ord}=\operatorname{ord}_{q}(2), m_{0}=\# G_{13 \cdot 17 \cdot q}=\phi(13 \cdot 17 \cdot q)=192(q-1) \\
m_{1}=\operatorname{Lcm}\left(\operatorname{ord}_{13}(2), \operatorname{ord}_{17}(2), \operatorname{ord}_{q}(2)\right)=\operatorname{Lcm}\left(24, \operatorname{ord}_{q}(2)\right)
\end{gathered}
$$

$u$ and $v$ are defined by (5.7) with $Q=13 \cdot 17 \cdot q$, thus $\tau_{Q}=v /(u+v)$.

5.3. EFFECT OF THE UNIVERSAL SEQUENCE $\mathcal{S}$ Let

$$
\mathcal{S}_{j}=\left\{q_{1}, q_{2}, \ldots, q_{j}\right\}
$$

be sub-sequences of the first $j$ elements of $\mathcal{S}$. Define

$$
\begin{aligned}
& y_{j}^{-}=\#\left\{\text { odd } h=15 t<10^{8}: j\right. \text { is the smallest subscript } \\
& \text { such that } \left.\mathcal{W}_{h}^{-}=\mathcal{S}_{j} \text { solves Problem } 2.1\right\} \text {, } \\
& y_{j}^{+}=\#\left\{\text { odd } h=15 t<10^{8}: j\right. \text { is the smallest subscript } \\
& \text { such that } \left.\mathcal{W}_{h}^{+}=\mathcal{S}_{j} \text { solves Problem 2.1 }\right\} \text {, } \\
& Y_{j}^{-}=\sum_{k=1}^{j} y_{k}^{-}, \text {and } Y_{j}^{+}=\sum_{k=1}^{j} y_{k}^{+} \text {. }
\end{aligned}
$$

Let $\mathcal{A}^{-}(h, \mathcal{S}), \mathcal{A}^{+}(h, \mathcal{S}), g^{-}(h, \mathcal{S})$ and $g^{+}(h, \mathcal{S})$ be as defined by $(5.9)$ and $(5.11)$ with

$$
L=\operatorname{Lcm}\left(\operatorname{ord}_{q_{1}}(2), \operatorname{ord}_{q_{2}}(2), \ldots, \operatorname{ord}_{q_{18}}(2)\right)=1034115765883200 .
$$

We use the following Procedure 5.3 to find, for each odd $h=15 t<10^{8}$, the smallest $j$ such that $\mathcal{W}_{h}=S_{j}$ solves Problem 2.1 and use Procedure 5.4 to find values of functions $y_{j}$ and $Y_{j}$, where $\mathcal{W}_{h}$ means either $\mathcal{W}_{h}^{-}$or $\mathcal{W}_{h}^{+}, y_{j}$ means either $y_{j}^{-}$or $y_{j}^{+}$, and $Y_{j}$ means either $Y_{j}^{-}$or $Y_{j}^{+}$. 
Procedure 5.3. Finding the smallest $j$ such that $\mathcal{W}_{h}=\mathcal{S}_{j}$ solves Problem 2.1;

\{input odd $h=15 t<10^{8}$ and the universal sequence $\mathcal{S}=\left\{q_{1}, \ldots, q_{48}\right\}$ \}

\{output $1 \leqslant j \leqslant 48$ such that $\mathcal{W}_{h}=\mathcal{S}_{j}$ solves Problem 2.1 \}

$\left\{\right.$ if $\mathcal{W}_{h}=\mathcal{S}_{48}=\mathcal{S}$ does not solve Problem 2.1, then output $\left.g(h, S)\right\}$

Begin $j \leftarrow 0$;

Repeat $j \leftarrow j+1$; Using Corollary 3.1 and Lemma 4.1 to find solutions in $n$ $\bmod 2 \operatorname{ord}_{q}(2)$ of $(2.4)$ for $q=q_{j}$;

If no solutions are found Then

begin output $j$ and the message " $q=q_{j}$ is a minus (plus) flag of $h$ "; exit end;

Using the Chinese Remainder Theorem to find common solutions in $n$ of the system (2.4) for all $q \in \mathcal{S}_{j}$;

Saving all solutions $\left.\bmod 2 \mathrm{Lcm} \operatorname{ord}_{q_{1}}, \ldots, \operatorname{ord}_{q_{j}}\right)$ in an array if they exist

Until $(j=48)$ Or (no common solutions for all $q \in \mathcal{S}_{j}$ exist);

Output $j$;

If $\mathcal{W}_{h}=\mathcal{S}_{48}=\mathcal{S}$ does not solve Problem 2.1 Then output $g(h, S)$

End;

Procedure 5.4. Finding values of functions $y_{j}$ and $Y_{j}$;

Begin $h \leftarrow 15$; For $k:=1$ To 48 Do begin $y_{k} \leftarrow 0$; $Y_{k} \leftarrow 0$ end;

Repeat Using Procedure 5.3 to find the smallest $j$ such that $\mathcal{W}_{h}=\mathcal{S}_{j}$ solves

Problem 2.1 or to find $g(h, S)$;

If the smallest $j$ is found Then

begin $y_{j} \leftarrow y_{j}+1$; For $k:=j$ To 48 Do $Y_{k} \leftarrow Y_{k}+1$ end;

$h \leftarrow h+30$

Until $h>10^{8}$;

For $k:=1$ To 48 Do output $y_{k}$ and $Y_{k}$

End.

In Table 4 we tabulate $y_{j}^{-}, y_{j}^{+}, Y_{j}^{-}, Y_{j}^{+}, Y_{j}^{-} /$Total and $Y_{j}^{+} /$Total where

$$
\text { Total }=\#\left\{\text { odd } h=15 t<10^{8}\right\}=3333333 \text {. }
$$

In Table 5 we tabulate all six odd $h=15 t$, all of them are of the form $h=4^{2 m}-1$, such that $\mathcal{S}$ has no subsets $\mathcal{W}_{h}^{-}$solving Problem 2.1. In Table 6 we tabulate all 52 odd $h=15 t$ such that $\mathcal{S}$ has no subsets $\mathcal{W}_{h}^{+}$solving Problem 2.1 , where $\# \mathcal{A}^{+}$means $\# \mathcal{A}^{+}(h, \mathcal{S})$. From Tables 4-6, one may clearly see the effect of the universal sequence $\mathcal{S}$.

REMARK 5.4. Since six $h=15 t$ tabulated in Table 5 are all of the form $4^{2 m}-1$, it seems that Problem 2.1 has finite solutions $\mathcal{W}_{h}^{-}$for all $h=15 t$ not of the form $4^{2 m}-1$. 
Table 2: Primes $q$ and $p$ with $\tau_{p q}>0$

\begin{tabular}{|c|c|c|c|c|c|c|c|c|}
\hline$q$ & $r$ & $p_{0}$ & $m_{0}$ & $m_{1}$ & $u$ & $v$ & $v /(u+v)$ & $p$ other than $p_{0}$ \\
\hline 17 & 1 & 13 & 192 & 24 & 4 & 4 & 0.50000 & \\
\hline 37 & 1 & 13 & 432 & 36 & 9 & 3 & 0.25000 & \\
\hline 41 & 2 & 17 & 640 & 40 & 11 & 5 & 0.31250 & 13 \\
\hline 61 & 1 & 41 & 2400 & 60 & 38 & 2 & 0.05000 & \\
\hline 73 & 2 & 13 & 864 & 36 & 20 & 4 & 0.16666 & 37 \\
\hline 97 & 2 & 17. & 1536 & 48 & 27 & 5 & 0.15625 & 13 \\
\hline 109 & 4 & 13 & 1296 & 36 & 29 & 7 & 0.19444 & $17,37,73$ \\
\hline 113 & 2 & 29 & 3136 & 28 & 97 & 15 & 0.13392 & 17 \\
\hline 157 & 1 & 53 & 8112 & 52 & 155 & 1 & 0.00641 & \\
\hline 193 & 2 & 17 & 3072 & 96 & 31 & 1 & 0.03125 & 97 \\
\hline 241 & 6 & 13 & 2880 & 24 & 87 & 33 & 0.27500 & $17,73,97,109,193$ \\
\hline 257 & 8 & 17 & 4096 & 16 & 143 & 113 & 0.44140 & $13,41,97,109,113,193,241$ \\
\hline 337 & 5 & 73 & 24192 & 63 & 357 & 27 & 0.07031 & $13,29,113,241$ \\
\hline 353 & 3 & 17 & 5632 & 88 & 63 & 1 & 0.01562 & 89,257 \\
\hline 397 & 4 & 89 & 34848 & 44 & 730 & 62 & 0.07828 & $17,257,353$ \\
\hline 433 & 6 & 73 & 31104 & 72 & 406 & 26 & 0.06018 & $17,37,109,241,257$ \\
\hline 457 & 3 & 17 & 7296 & 152 & 47 & 1 & 0.02083 & 229,257 \\
\hline 577 & 3 & 17 & 9216 & 144 & 63 & 1 & 0.01562 & 241,257 \\
\hline 601 & 1 & 101 & 60000 & 100 & 594 & 6 & 0.01000 & \\
\hline 641 & 3 & 257 & 163840 & 64 & 2459 & 101 & 0.03945 & 17,97 \\
\hline 673 & 7 & 17 & 10752 & 48 & 202 & 22 & 0.09821 & $13,97,109,193,241,257$ \\
\hline 881 & 2 & 89 & 77440 & 55 & 1317 & 91 & 0.06463 & 601 \\
\hline 937 & 1 & 73 & 67392 & 117 & 567 & 9 & 0.01562 & \\
\hline 953 & 1 & 137 & 129472 & 68 & 1887 & 17 & 0.00892 & \\
\hline 1013 & 1 & 277 & 279312 & 92 & 3029 & 7 & 0.00230 & \\
\hline 1217 & 2 & 17 & 19456 & 152 & 127 & 1 & 0.00781 & 257 \\
\hline 1321 & 8 & 41 & 52800 & 60 & 851 & 29 & 0.03295 & $13,17,61,73,241,257,337$ \\
\hline 1429 & 4 & 13 & 17136 & 84 & 202 & 2 & 0.00980 & $29,113,337$ \\
\hline 1613 & 5 & 157 & 251472 & 52 & 4690 & 146 & 0.03019 & $17,53,257,1249$ \\
\hline 1657 & 2 & 277 & 457056 & 92 & 4956 & 12 & 0.00241 & 1013 \\
\hline 1777 & 1 & 593 & 1051392 & 148 & 7103 & 1 & 0.00014 & \\
\hline 1801 & 6 & 601 & 1080000 & 25 & 34352 & 8848 & 0.20481 & $41,101,401,881,1321$ \\
\hline 2089 & 2 & 233 & 484416 & 29 & 14035 & 2669 & 0.15978 & 349 \\
\hline 2113 & 6 & 89 & 185856 & 44 & 3849 & 375 & 0.08877 & $17,257,353,397,881$ \\
\hline 2593 & 1 & 73 & 186624 & 81 & 2258 & 46 & 0.01996 & \\
\hline
\end{tabular}


Table 3: The Universal Sequence $\mathcal{S}=\left\{q_{1}, q_{2}, \cdots, q_{48}\right\}$

\begin{tabular}{|c|c|c|c|c|c|c|c|c|}
\hline$j$ & $q=q_{j}$ & $\pi_{q}$ & ord & $m_{0}$ & $m_{1}$ & $u$ & $v$ & $\frac{v}{u+v}$ \\
\hline 1 & 13 & $3+2 i$ & 12 & & & & & \\
\hline 2 & 17 & $1+4 i$ & 8 & & & & & \\
\hline 3 & 241 & $-15+4 i$ & 24 & 46080 & 24 & 330 & 1590 & 0.82812 \\
\hline 4 & 97 & $9+4 i$ & 48 & 18432 & 48 & 102 & 282 & 0.73437 \\
\hline 5 & 257 & $1+16 i$ & 16 & 49152 & 48 & 286 & 738 & 0.72070 \\
\hline 6 & 673 & $-23+12 i$ & 48 & 129024 & 48 & 765 & 1923 & 0.71540 \\
\hline 7 & 37 & $-1+6 i$ & 36 & 6912 & 72 & 32 & 64 & 0.66666 \\
\hline 8 & 109 & $3+10 i$ & 36 & 20736 & 72 & 98 & 190 & 0.65972 \\
\hline 9 & 41 & $5+4 i$ & 20 & 7680 & 120 & 22 & 42 & 0.65625 \\
\hline 10 & 73 & $-3+8 i$ & 9 & 13824 & 72 & 66 & 126 & 0.65625 \\
\hline 11 & 433 & $17+12 i$ & 72 & 82944 & 72 & 419 & 733 & 0.63628 \\
\hline 12 & 193 & $-7+12 i$ & 96 & 36864 & 96 & 146 & 238 & 0.61979 \\
\hline 13 & 1321 & $5+36 i$ & 60 & 253440 & 120 & 916 & 1196 & 0.56628 \\
\hline 14 & 61 & $-5+6 i$ & 60 & 11520 & 120 & 42 & 54 & 0.56250 \\
\hline 15 & 577 & $1+24 i$ & 144 & 110592 & 144 & 337 & 431 & 0.56119 \\
\hline 16 & 337 & $9+16 i$ & 21 & 64512 & 168 & 178 & 206 & 0.53645 \\
\hline 17 & 1429 & $23+30 i$ & 84 & 274176 & 168 & 764 & 868 & 0.53186 \\
\hline 18 & 113 & $-7+8 i$ & 28 & 21504 & 168 & 60 & 68 & 0.53125 \\
\hline 19 & 397 & $19+6 i$ & 44 & 76032 & 264 & 136 & 152 & 0.52777 \\
\hline 20 & 1249 & $-15+32 i$ & 156 & 239616 & 312 & 376 & 392 & 0.51041 \\
\hline 21 & 353 & $17+8 i$ & 88 & 67584 & 264 & 126 & 130 & 0.50781 \\
\hline 22 & 2113 & $33+32 i$ & 44 & 405504 & 264 & 762 & 774 & 0.50390 \\
\hline 23 & 29 & $-5+2 i$ & 28 & 5376 & 168 & 16 & 16 & 0.50000 \\
\hline 24 & 53 & $7+2 i$ & 52 & 9984 & 312 & 16 & 16 & 0.50000 \\
\hline 25 & 101 & $-1+10 i$ & 100 & 19200 & 600 & 16 & 16 & 0.50000 \\
\hline 26 & 157 & $11+6 i$ & 52 & 29952 & 312 & 48 & 48 & 0.50000 \\
\hline 27 & 233 & $13+8 i$ & 29 & 44544 & 696 & 32 & 32 & 0.50000 \\
\hline 28 & 277 & $-9+14 i$ & 92 & 52992 & 552 & 48 & 48 & 0.50000 \\
\hline 29 & 349 & $-5+18 i$ & 348 & 66816 & 696 & 48 & 48 & 0.50000 \\
\hline 30 & 401 & $1+20 i$ & 200 & 76800 & 600 & 64 & 64 & 0.50000 \\
\hline 31 & 881 & $25+16 i$ & 55 & 168960 & 1320 & 64 & 64 & 0.50000 \\
\hline 32 & 937 & $-19+24 i$ & 117 & 179712 & 936 & 96 & 96 & 0.50000 \\
\hline 33 & 1013 & $23+22 i$ & 92 & 194304 & 552 & 176 & 176 & 0.50000 \\
\hline 34 & 1657 & $-19+36 i$ & 92 & 317952 & 552 & 288 & 288 & 0.50000 \\
\hline 35 & 2089 & $45+8 i$ & 29 & 400896 & 696 & 288 & 288 & 0.50000 \\
\hline 36 & 89 & $5+8 i$ & 11 & 16896 & 264 & 32 & 32 & 0.50000 \\
\hline 37 & 137 & $-11+4 i$ & 68 & 26112 & 408 & 32 & 32 & 0.50000 \\
\hline 38 & 229 & $15+2 i$ & 76 & 43776 & 456 & 48 & 48 & 0.50000 \\
\hline 39 & 457 & $21+4 i$ & 76 & 87552 & 456 & 94 & 98 & 0.51041 \\
\hline 40 & 593 & $-23+8 i$ & 148 & 113664 & 888 & 64 & 64 & 0.50000 \\
\hline 41 & 601 & $5+24 i$ & 25 & 115200 & 600 & 96 & 96 & 0.50000 \\
\hline 42 & 641 & $25+4 i$ & 64 & 122880 & 192 & 308 & 332 & 0.51875 \\
\hline 43 & 953 & $13+28 i$ & 68 & 182784 & 408 & 224 & 224 & 0.50000 \\
\hline 44 & 1217 & $-31+16 i$ & 152 & 233472 & 456 & 254 & 258 & 0.50390 \\
\hline 45 & 1613 & $-13+38 i$ & 52 & 309504 & 312 & 494 & 498 & 0.50201 \\
\hline 46 & 1777 & $-39+16 i$ & 74 & 340992 & 888 & 192 & 192 & 0.50000 \\
\hline 47 & 1801 & $-35+24 i$ & 25 & 345600 & 600 & 288 & 288 & 0.50000 \\
\hline 48 & 2593 & $17+48 i$ & 81 & 497664 & 648 & 384 & 384 & 0.50000 \\
\hline
\end{tabular}


REMARK 5.5. It is easy to prove that, if $h=15 t$ is of the form $4^{2 m}-1$, then for any finite set of primes $\left\{q_{j}\right\}$, the system (5.3) has solutions in $n$. It seems that the gcd checking in (2.2) and (2.3) does not help much for $h$ of this form. So, it seems that Problem 2.1 does not have a finite solution $\mathcal{W}_{h}$ for $h=15 t$ of the form $4^{2 m}-1$. Nevertheless, the determination of values of the functions $g(h, \mathcal{S})$ (see Tables 5 and 6) is a remediation of finite-solution-non-existing for $h=15 t$ of the form $4^{2 m}-1$. However, Bosma's approach for the case $h \equiv 0 \bmod 3$ did not provide such remediation.

REMARK 5.6. Out of the 52 odd $h=15 t$ tabulated in Table 6 , there are 46 odd $h=15 t$ not of the form $4^{2 m}-1$. Although Problem 2.1 does not have solutions $\mathcal{W}_{h}^{+} \subseteq \mathcal{S}$ for these 46 odd $h=15 t_{2}$ it seems that for all odd $h=15 t$ not of the form $4^{2 m}-1$, Problem 2.1 has finite solutions $\mathcal{W}_{h}^{+}$with additional primes outside $\mathcal{S}$. For examples,

$$
\begin{aligned}
\mathcal{W}_{76245}^{+} & =\{29,37,61,73,277,1289,1657\} \subset \mathcal{S} \cup\{1289\} \subset \mathcal{P}, \\
\mathcal{W}_{794155}^{+} & =\{13,29,73,281\} \subset \mathcal{S} \cup\{281\} \subset \mathcal{P} \\
\mathcal{W}_{1259445}^{+} & =\{13,37,97,353,7393\} \subset \mathcal{S} \cup\{7393\} \subset \mathcal{P} \cup\{7393\}
\end{aligned}
$$

solve Problem 2.1 for corresponding $h$.

REMARK 5.7. Note that, using (1.8) to test primality of $M_{h, n,-}$ for the case $h \equiv 0 \bmod 3$, one first finds $d \in \mathbb{Z}$ satisfying (1.6), then he finds an algebraic integer $\alpha$ in the quadratic extension $\mathbb{Q}(\sqrt{d})$ satisfying (1.7). Moreover, for determining finite judge set (1.13), Bosma needed the complete factorisations of all integers $2^{u}-1$ for $2 \leqslant u \leqslant U=250$. So, the judge $(d, \alpha)$ in Bosma's approach is not only "two-process finding", but also $d$ may be as large as up to 106 decimal digits. In our generalised test, judges are "one-process finding", which are just some small primes $\equiv 1 \bmod 4$. Moreover we have the universal sequence $\mathcal{S}$ of judges for odd $h=15 t<10^{8}$, but Bosma did not provide such a sequence of judges for odd $h=3 t<10^{5}$.

REMARK 5.8. Now one may realise that the bound 2600 for $p$ and the bound 350 for ord $p(2)$ in the set $\mathcal{P}$ defined by (4.1) are chosen after several trials for obtaining the effective universal sequence $\mathcal{S}$ of judges for odd $h=15 t<10^{8}$. One may increase these bounds for $h>10^{8}$.

REMARK 5.9. For determining the values of $g^{+}(h, S)$ defined by $(5.11)$, one needs to check whether $M_{h, n,+}$ is a perfect square. This can be done by Newton's method (see [3, Section 1.7] and [4, Section 9.2.2]) or by Bosma [2, Proposition 4.2].

Remark 5.10. We call $\mathcal{W}_{h}$ a minimal set of judges to solve Problem 2.1 for given odd $h$, if any proper subset of $\mathcal{W}_{h}$ does not solve Problem 2.1 but $\mathcal{W}_{h}$ itself solves Problem 2.1. Note that $\mathcal{W}_{h}$ means either $\mathcal{W}_{h}^{-}$or $\mathcal{W}_{h}^{+}$. The subsequence $\mathcal{S}_{j}$ found by Procedure 5.3 is in general not a minimal set of judges to solve Problem 2.1 for given odd $h=15 t$. One may delete some elements of $\mathcal{S}_{j}$ to obtain a minimal set $\mathcal{W}_{h}$. For example, the subsequence $\mathcal{S}_{j}$ found by Procedure 5.3 for $h=11897535=15 \cdot 793169$ is $\mathcal{W}_{h}^{-}=\mathcal{S}_{34}$. But both $\mathcal{W}_{h}^{-}=\{277,1013,1657\} \subset \mathcal{S}_{34}$ and $\mathcal{W}_{h}^{-}=\{229,277,457,593,1657,1777\} \subset \mathcal{S}_{46}$ are minimal sets solving Problem 2.1 for $h=11897535$. So, there may exist several minimal sets (with different cardinalities) of judges to solve Problem 2.1 for given odd $h$. 
Table 4: The functions $y_{j}^{-}, y_{j}^{+}, Y_{j}^{-}, Y_{j}^{+}, \frac{Y_{j}^{-}}{\text {Total }}$ and $\frac{Y_{j}^{+}}{\text {Total }}$

\begin{tabular}{|c|c|c|c|c|c|c|c|}
\hline$j$ & $q_{j}$ & $y_{j}^{-}$ & $Y_{j}^{-}$ & $\frac{Y_{j}^{-}}{\text {Total }}$ & $y_{j}^{+}$ & $Y_{j}^{+}$ & $\frac{Y_{j}^{+}}{\text {Total }}$ \\
\hline 1 & 13 & 429 & 429 & 0.00012 & 429 & 429 & 0.00012 \\
\hline 2 & 17 & 1554395 & 1554824 & 0.46644 & 1554395 & 1554824 & 0.46644 \\
\hline 3 & 241 & 1049748 & 2604572 & 0.78137 & 1049752 & 2604576 & 0.78137 \\
\hline 4 & 97 & 384445 & 2989017 & 0.89670 & 384927 & 2989503 & 0.89685 \\
\hline 5 & 257 & 240217 & 3229234 & 0.96877 & 239660 & 3229163 & 0.96874 \\
\hline 6 & 673 & 76507 & 3305741 & 0.99172 & 76503 & 3305666 & 0.99169 \\
\hline 7 & 37 & 11892 & 3317633 & 0.99528 & 12124 & 3317790 & 0.99533 \\
\hline 8 & 109 & 11098 & 3328731 & 0.99861 & 10966 & 3328756 & 0.99862 \\
\hline 9 & 41 & 1823 & 3330554 & 0.99916 & 1776 & 3330532 & 0.99915 \\
\hline 10 & 73 & 2013 & 3332567 & 0.99977 & 1985 & 3332517 & 0.99975 \\
\hline 11 & 433 & 566 & 3333133 & 0.99993 & 497 & 3333014 & 0.99990 \\
\hline 12 & 193 & 95 & 3333228 & 0.99996 & 122 & 3333136 & 0.99994 \\
\hline 13 & 1321 & 60 & 3333288 & 0.99998 & 66 & 3333202 & 0.99996 \\
\hline 14 & 61 & 19 & 3333307 & 0.99999 & 15 & 3333217 & 0.99996 \\
\hline 15 & 577 & 8 & 3333315 & 0.99999 & 5 & 3333222 & 0.99996 \\
\hline 16 & 337 & 0 & 3333315 & 0.99999 & 2 & 3333224 & 0.99996 \\
\hline 17 & 1429 & 7 & 3333322 & 0.99999 & 11 & 3333235 & 0.99997 \\
\hline 18 & 113 & 0 & 3333322 & 0.99999 & 2 & 3333237 & 0.99997 \\
\hline 19 & 397 & 0 & 3333322 & 0.99999 & 1 & 3333238 & 0.99997 \\
\hline 20 & 1249 & 0 & 3333322 & 0.99999 & 2 & 3333240 & 0.99997 \\
\hline 21 & 353 & 1 & 3333323 & 0.99999 & 9 & 3333249 & 0.99997 \\
\hline 22 & 2113 & 1 & 3333324 & 0.99999 & 2 & 3333251 & 0.99997 \\
\hline 23 & 29 & 0 & 3333324 & 0.99999 & 1 & 3333252 & 0.99997 \\
\hline 24 & 53 & 1 & 3333325 & 0.99999 & 5 & 3333257 & 0.99997 \\
\hline 25 & 101 & 0 & 3333325 & 0.99999 & 2 & 3333259 & 0.99997 \\
\hline 26 & 157 & 0 & 3333325 & 0.99999 & 5 & 3333264 & 0.99997 \\
\hline 27 & 233 & 0 & 3333325 & 0.99999 & 0 & 3333264 & 0.99997 \\
\hline 28 & 277 & 0 & 3333325 & 0.99999 & 0 & 3333264 & 0.99997 \\
\hline 29 & 349 & 1 & 3333326 & 0.99999 & 2 & 3333266 & 0.99997 \\
\hline 30 & 401 & 0 & 3333326 & 0.99999 & 1 & 3333267 & 0.99998 \\
\hline 31 & 881 & 0 & 3333326 & 0.99999 & 2 & 3333269 & 0.99998 \\
\hline 32 & 937 & 0 & 3333326 & 0.99999 & 3 & 3333272 & 0.99998 \\
\hline 33 & 1013 & 0 & 3333326 & 0.99999 & 1 & 3333273 & 0.99998 \\
\hline 34 & 1657 & 1 & 3333327 & 0.99999 & 4 & 3333277 & 0.99998 \\
\hline 35 & 2089 & 0 & 3333327 & 0.99999 & 4 & 3333281 & 0.99998 \\
\hline $36-48$ & $89-2593$ & 0 & 3333327 & 0.99999 & 0 & 3333281 & 0.99998 \\
\hline
\end{tabular}


Table 5: All odd $h=15 t<10^{8}$ such that $\mathcal{S}$ does not have subsets $\mathcal{W}_{h}^{-}$solving Problem 2.1

\begin{tabular}{|r|r|r|r|}
\hline$t$ & $h=15 t$ & $\# \mathcal{A}^{-}(h, \mathcal{S})$ & $g^{-}(h, \mathcal{S})$ \\
\hline 1 & 15 & 128 & 18632716502396 \\
17 & 255 & 32 & 44961555038392 \\
273 & 4095 & 144 & 2374757985588 \\
4369 & 65535 & 12 & 392017741315184 \\
69905 & 1048575 & 96 & 18295167290380 \\
1118481 & 16777215 & 24 & 124218110015 \\
\hline
\end{tabular}

Table 6: All odd $h=15 t<10^{8}$ such that $\mathcal{S}$ does not have subsets $\mathcal{W}_{h}^{+}$solving Problem 2.1

\begin{tabular}{|c|c|c|c|c|c|c|c|}
\hline$t$ & $h=15 t$ & $\# A^{+}$ & $g^{+}(h, \mathcal{S})$ & $t$ & $h=15 t$ & $\# A^{+}$ & $g^{+}(h, s)$ \\
\hline 1 & 15 & 384 & 3744810669600 & 1555421 & 23331315 & 240 & 9316358251204 \\
\hline 17 & 255 & 80 & 32874965323200 & 1620409 & 24306135 & 16 & 208796029041605 \\
\hline 273 & 4095 & 192 & 27949074753600 & 1624207 & 24363105 & 96 & 21416915519980 \\
\hline 4369 & 65535 & 216 & 35892230774400 & 1689743 & 25346145 & 72 & 79343920655984 \\
\hline 5083 & 76245 & 96 & 14312086588806 & 1693839 & 25407585 & 72 & 57450875882388 \\
\hline 52941 & 794115 & 96 & 56017284523205 & 1694095 & 25411425 & 48 & 9322657343992 \\
\hline 69905 & 1048575 & 128 & 13517853148800 & 1694111 & 25411665 & 24 & 101383898615996 \\
\hline 83963 & 1259445 & 540 & 1245274430404 & 1737631 & 26064465 & 400 & 7826125507172 \\
\hline 197743 & 2966145 & 96 & 13335179457604 & 2045407 & 30681105 & 20 & 118007204515176 \\
\hline 224927 & 3373905 & 128 & 16007983991976 & 2053685 & 30805275 & 16 & 419236121304004 \\
\hline 234481 & 3517215 & 16 & 157830069196805 & 2801779 & 42026685 & 16 & 172352627647200 \\
\hline 298497 & 4477455 & 144 & 12966968851200 & 2941299 & 44119485 & 144 & 44977439707204 \\
\hline 325039 & 4875585 & 30 & 24573582633588 & 3093983 & 46409745 & 432 & 4566842279980 \\
\hline 346579 & 5198685 & 288 & 1048798951208 & 3159519 & 47392785 & 120 & 730694764784 \\
\hline 546165 & 8192475 & 12 & 46581791256004 & 3163615 & 47454225 & 96 & $2173816925278 B$ \\
\hline 575631 & 8634465 & 24 & 148696384636776 & 3163871 & $\$ 7458065$ & 60 & 5884015737592 \\
\hline 733907 & 11008605 & 96 & 16897316436005 & 3163887 & 47458305 & 320 & 5388873890396 \\
\hline 807823 & 12117345 & 8 & 225215774783996 & 3267165 & 49007475 & 450 & 1177930353605 \\
\hline 1118481 & 16777215 & 16 & 37586686737600 & 3398759 & 50981385 & 16 & 27035706297606 \\
\hline 1227083 & 18406245 & 128 & 5401061265604 & 3732271 & 55984085 & 72 & 31697034969604 \\
\hline 1273503 & 19102545 & 288 & 7826125507180 & 4277607 & 64164105 & 144 & 13974537376804 \\
\hline 1288007 & 19320105 & 72 & 31054527504007 & 4472013 & 67080195 & 144 & 6210905500804 \\
\hline 1339039 & 20085585 & 32 & 24843622003184 & 5589327 & 83839905 & 32 & 47034504316772 \\
\hline 1343135 & 20147025 & 40 & 87866045467188 & 5618313 & 84274695 & 240 & 14921556249606 \\
\hline 1343391 & 20150865 & 96 & 45189691747192 & 6275577 & 94133655 & 60 & 37448106696004 \\
\hline 1343407 & 20151105 & 144 & 1473987715196 & 6384911 & 95773665 & 16 & 97416702583176 \\
\hline
\end{tabular}

\section{Conclusions}

We have presented a Lucasian primality test mainly for numbers of the form $M_{h, n}=h \cdot 2^{n} \pm 1$ with odd $h=15 t<2^{n-2}-1$, which generalises Berrizbeitia and Berry's test for such numbers with $h \not \equiv 0 \bmod 5$. With our generalised test, the primality of such a number $M_{h, n}$ can be proved by means of a Lucas sequence with a seed determined by $h$ and $\pi_{q}$ - primary irreducible divisor of a prime $q \equiv 1 \bmod 4$. We call the prime $q$ a judge of the number $M_{h, n}$.

Given an odd $h$, if the numbers $M_{h, n,-}=h \cdot 2^{n}-1$ (respectively, $M_{h, n,+}=h \cdot 2^{n}+1$ ) share a common judge $q$ for all $n$, we call the judge $q$ a minus (respectively plus) flag of $h$. We found sixteen primes $q$ which are minus or plus flags of some odd $h=15 t<10^{8} ; 298217$ (about $8.9465 \%$ ) out of 3333333 odd $h=15 t<10^{8}$ have minus flags, and 298237 (about $8.9471 \%$ ) odd $h=15 t<10^{8}$ have plus flags. 
We have also prescribed a sequence $\mathcal{S}$ of 48 primes $\equiv 1 \bmod 4$ in the interval $[13,2593]$ including the sixteen flags, such that, for 3333327 out of the 3333333 odd $h=15 t<10^{8}$ and for all $n$, each number $M_{h, n,-}$ has a judge $q$ in the first 34 elements of $\mathcal{S}$. For the remaining six $h$ (which are of the form $4^{2 m}-1$ ) and for all $n<2.37 \cdot 10^{12}$, each number $M_{h, n,-}$ has a judge $q$ in $\mathcal{S}$. Correspondent words can be said for the numbers $M_{h, n,+}=h \cdot 2^{n}+1$. For 3333281 out of the 3333333 odd $h=15 t<10^{8}$ and for all $n$, each number $M_{h, n,+}$ has a judge $q$ in the first 35 elements of $\mathcal{S}$. For the remaining fifty two $h$ and for all $n<7.3 \cdot 10^{11}$, each number $M_{h, n,+}$ has a judge $q$ in $\mathcal{S}$. The only limitation towards testing primality of numbers $M_{h, n}$ by our generalised test seems to be the difficulty of doing computation involving such numbers as large as

$$
4095 \cdot 2^{2374757985588}-1 \text { or } 47392785 \cdot 2^{730694764784}+1 \text {, }
$$

much larger than the 43rd Mersenne prime (current largest known prime number)

$$
2^{30402457}-1 \text { (9152052 decimal digits), }
$$

see http://www.mersenne.org, [5, A3], and [9, Chapter 5].

\section{REFERENCES}

[1] P. Berrizbeitia and T.G. Berry, 'Biquadratic reciprocity and a Lucasian primality test', Math. Comp. 73 (2004), 1559-1564.

[2] W. Bosma, 'Explicit primality criteria for $h \cdot 2^{n} \pm 1$ ', Math. Comp. 61 (1993), 97-109.

[3] H. Cohen, A course in computational algebraic number theory, Graduate Texts in Mathmatics 138 (Springer-Verlag, Berlin, 1996).

[4] R. Crandall and C. Pomerance, Prime numbers, a computational perspective (Springer-Verlag, New York, 2005).

[5] R.K. Guy, Unsolved problems in number theory (Springer-Verlag, New York, 2004).

[6] K. Ireland and M. Rosen, A classical introduction to modern number theory, Graduate Texts in Mathematics 84 (Springer-Verlag, New York, 1990).

[7] D.H. Lehmer, 'On Lucas's test for the primality of Mersenne's number', J. London Math. Soc. 10 (1935), 162-165.

[8] E. Lucas, 'Théorie des fonctions numériques simplement périodiques, I. II', Amer. J. Math. 1 (1878), 184-239, 289-321.

[9] P. Ribenboim, The little book of bigger primes (Springer-Verlag, New York, 2004).

[10] H. Riesel, 'Lucasian criteria for the primality of $N=h \cdot 2^{n}-1$ ', Math. Comp. 23 (1969), 869-875.

[11] H. Riesel, Prime numbers and computer methods for factorization (Birkhaüser, Boston, 1985).

[12] Z. Zhang, 'Finding strong pseudoprimes to several bases', Math. Comp. 70 (2001), 863-872. 
Department of Mathematics

Anhui Normal University

Wuhu 241000

Anhui

Peoples Republic of China

e-mail: zhangzhx@mail.wh.ah.cn

Department of Applied Mathematics

Anhui University of Finance \& Economics

Bengbu 233041

Anhui

Peoples Republic of China

e-mail: liuxianbei82@163.com
Department of Mathematics

Anqing Teachers College

Anqing 246011

Anhui

Peoples Republic of China

e-mail: pingzi212@163.com 